\title{
Some properties of locally conformal symplectic structures
}

\author{
Augustin Banyaga
}

\begin{abstract}
We show that the $d_{\omega}$-cohomology is isomorphic to a conformally invariant usual de Rham cohomology of an appropriate cover. We also prove a Moser theorem for locally conformal symplectic (lcs) forms. We point out a connection between lcs geometry and contact geometry. Finally, we show the connections between first kind, second kind, essential, inessential, local, and global conformal symplectic structures through several invariants.
\end{abstract}

Mathematics Subject Classification (2000). 53C12; 53C15.

Keywords. Locally conformal symplectic structures, Lee form, extended Lee homomorphism, de Rham invariant, Gelfand-Fucks invariant, Lee invariant, conformal invariants, essential/inessential conformal structures, the $d_{\omega}$ cohomology, the $c \mathcal{A}$-cohomology.

\section{Preliminaries}

A locally conformal symplectic (lcs) form on a smooth manifold $M$ is a nondegenerate 2 -form $\Omega$ such that there exists an open cover $\mathcal{U}=\left(U_{i}\right)$ and smooth positive functions $\lambda_{i}$ on $U_{i}$ such that

$$
\Omega_{i}=\lambda_{i}\left(\Omega_{\mid U_{i}}\right)
$$

is a symplectic form on $U_{i}$. If for all $i, \lambda_{i}=1$, the form $\Omega$ is a symplectic form. Lee [15] observed that the 1-forms $\left\{d\left(\ln \lambda_{i}\right)\right\}$ fit together into a closed 1-form $\omega$ such that

$$
d \Omega=-\omega \wedge \Omega .
$$

Such 1-form is uniquely determined by $\Omega$ and is called the Lee form of $\Omega$.

Conversely, if a non-degenerate 2 -form $\Omega$ satisfies $(1)$, and $\mathcal{U}=(U)_{i}$ is an open cover with contractible open sets, then $\omega_{\mid U_{i}}=d \ln \lambda_{i}$, for some positive function $\lambda_{i}$ on $U_{i}$ and $\lambda_{i} \Omega_{\mid U_{i}}$ is symplectic.

Two lcs forms $\Omega, \Omega^{\prime}$ on a smooth manifold $M$ are said to be (conformally) equivalent if $\Omega^{\prime}=f \Omega$, for some positive function $f$ on $M$.

A locally conformal symplectic (lcs) structure $\mathcal{S}$ on a smooth manifold $M$ is an equivalence class of lcs forms. 
The couple $(M, \mathcal{S})$ is called a lcs manifold. If $\Omega$ is a representative of $\mathcal{S}$, we write $\Omega \in \mathcal{S}$. If $\omega=0$ in the definition above, then $\Omega$ is a symplectic form. In that case the lcs structure $\mathcal{S}$ is said to be a global conformal symplectic (gcs) structure and we write $\mathcal{S}=\mathcal{O}$.

Let $(M, \mathcal{S})$ be a lcs manifold, and let $\Omega \in \mathcal{S}$ and $\omega$ its Lee form. If $\Omega^{\prime}=\lambda \Omega$ for some positive function $\lambda$, then an immediate calculation shows that the Lee form of $\Omega^{\prime}$ is $\omega^{\prime}=\omega-d \ln (\lambda)$.

Hence the cohomology class $[\omega] \in H^{1}(M, \mathbb{R})$ is an invariant $\mathcal{L}_{\mathcal{S}}$ of $\mathcal{S}$, we call the Lee class of $\mathcal{S}$. Clearly, $\mathcal{S}=\mathcal{O}$ iff $\mathcal{L}_{\mathcal{S}}=0$.

Locally conformal symplectic forms were introduced by Lee [15], and have been extensively studied by Vaisman [18], [19]. The first properties of their automorphism groups were established by Lefebvre [16].

We will assume that all manifolds considered are connected, but not necessarily compact, and have dimension at least 4. (In dimension 2, a lcs form is simply a volume-form, and the corresponding structure is an orientation.)

For any closed 1-form $\omega$ on a smooth manifold $M$, the operator $d_{\omega}$ which assigns to a $p$-form $\gamma$ the $(p+1)$-form

$$
d_{\omega} \gamma=d \gamma+\omega \wedge \gamma
$$

is a coboundary operator, i.e. $d_{\omega} \circ d_{\omega}=0$.

The cohomology of differential forms with this coboundary operator will be denoted by $H_{\omega}^{*}(M)$ and will be called the $d_{\omega}$-cohomology. For more information on this cohomology, see [11] or [19].

A lcs form $\Omega$ is precisely a non-degenerate $d_{\omega}$ closed 2 -form (where $\omega$ is the Lee form).

This cohomology is "almost" an invariant of the lcs structure $\mathcal{S}=[\Omega]$ : given $\Omega^{\prime} \in \mathcal{S}$, there is an isomorphism between $H_{\omega}(M)$ and $H_{\omega^{\prime}}(M)$, ( $\omega^{\prime}$ the Lee form of $\Omega^{\prime}$ ), depending on the choice of $\lambda$ such that $\omega^{\prime}=\omega-d \ln \lambda$. More precisely the isomorphism is given by $\alpha \mapsto \lambda \alpha$.

In section 3 , we show that the $c \mathcal{A}$ cohomology constructed in [5], [6], is isomorphic to $H_{\omega}(M)$. This shows that the $d_{\omega}$ cohomology (which is a sort of twisted de Rham cohomology of $M$ ) is a conformally invariant usual de Rham cohomology of an appropiate cover of $M$.

Let $\operatorname{Diff}_{\mathcal{S}}(M)$ be the group of all automorphisms of a lcs structure $\mathcal{S}$ on a smooth manifold $M$. It is clear that for any representative $\Omega \in \mathcal{S}$, then $\operatorname{Diff}_{\mathcal{S}}(M)$ is the set of all diffeomorphisms $\phi$ of $M$ such that $\phi^{*} \Omega=f_{\phi} \Omega$, where $f_{\phi}$ is a nowhere zero (positive) smooth function on $M$.

We also may choose (or fix) an underlying $\Omega \in \mathcal{S}$, and consider the group $G_{\Omega}(M)$ of diffeomorphisms of $M$ which preserve the form $\Omega$. This is a noninvariant subgroup of $\operatorname{Diff}_{\mathcal{S}}(M)$.

The Lie algebra $\mathcal{X}_{\mathcal{S}}(M)$ of infinitesimal automorphisms of $\mathcal{S}$, consists of vector fields $X$ on $M$ such that $L_{X} \Omega=\left(u_{\Omega}(X)\right) \Omega$, where $u_{\Omega}(X)$ is a smooth function on $M$. Here $L_{X}$ stands for the Lie derivative in the direction $X$. We denote $\mathcal{X}_{\mathcal{S}}(M)_{c}$ 
the subalgebra of compact supported automorphisms. We will also consider the subalgebra $\mathcal{X}_{\Omega}(M)$ of $\mathcal{X}_{\mathcal{S}}(M)$ consisting of vector fields $X$ such that $L_{X} \Omega=0$.

Definition. A lcs form $\Omega$ on $M$ is said to be of the first kind if there exists $X \in \mathcal{X}_{\Omega}(M)$, with $\omega(X) \neq 0$, where $\omega$ is the corresponding Lee form. Otherwise it is said to be of the second kind [18].

A lcs structure $\mathcal{S}$ on $M$ is said to be of the first kind if there is a representative $\Omega \in \mathcal{S}$ of the first kind. The lcs structure $\mathcal{S}$ is said to be of the second kind otherwise.

Warning. Vaisman [18] observed that a first kind lcs structure admits representatives which are second kind lcs forms.

For $X \in \mathcal{X}_{\Omega}(M)$, and $M$ connected, $\omega(X)$, is a constant number since:

$$
0=d L_{X} \Omega=L_{X} d \Omega=L_{X}(-\omega \wedge \Omega)=-\left(\left(L_{X} \omega\right) \wedge \Omega+\omega \wedge L_{X} \Omega\right)=-(\operatorname{di}(X) \omega) \wedge \Omega
$$

and $\Omega$ is non-degenerate.

Hence if $\Omega$ is a first kind lcs form with Lee form $\omega$, the condition:

$$
\text { There is } X \in \mathcal{X}_{\Omega}(M) \text {, with } \omega(X) \neq 0
$$

is equivalent to saying that there a 1 -form $\theta$ such that

$$
\Omega=d \theta+\omega \wedge \theta
$$

Indeed just normalize $X$ as above so that $\omega(X)=1$ and set $\theta=i(X) \Omega$. First kind lcs forms are $d_{\omega}$ exact.

\section{Examples}

We describe here a few examples of lcs forms. The reader can consult the book [9] for more examples.

\subsection{Examples connected with Contact Geometry}

A contact form $\alpha$ on a $(2 \mathrm{n}+1)$ dimensional manifold $N$ is a 1 -form $\alpha$ such that $\alpha \wedge(d \alpha)^{n}$ is everywhere non-zero. Two contact forms $\alpha$ and $\alpha^{\prime}$ are equivalent if there is a smooth positive function $f$ on $N$ such that $\alpha^{\prime}=f \alpha$. The contact structure $\mathcal{C}(\alpha)$, determined by $\alpha$ is the equivalence class of $\alpha$.

Consider the cartesian product $M=N \times S^{1}$, and the projections $p_{1}: M \rightarrow N$, $p_{2}: M \rightarrow S^{1}$. Let $\beta$ be the canonical 1-form on $S^{1}$ with integral 1. If we set $\theta=p_{1}^{*} \alpha$ and $\omega=p_{2}^{*} \beta$, then

$$
\Omega=d \theta+\omega \wedge \theta
$$


is non-degenerate and $d \Omega=-\omega \wedge d \theta=-\omega \wedge(\Omega-\omega \wedge \theta)=-\omega \wedge \Omega+\omega \wedge \omega \wedge \theta=-\omega \wedge \Omega$. Hence the conformal class of $\Omega$ is a lcs structure on $M$, we denote $\mathcal{S}(\alpha)$. This structure is of the first kind.

The following result will be proved in section 4 .

Theorem 1. The lcs structure $\mathcal{S}(\alpha)$ depends only on the contact structure $\mathcal{C}(\alpha)$. In fact there is a well defined mapping from the group $\operatorname{Diff}_{\mathcal{C}(\alpha)}(M)$ of automorphisms of the contact structure $\mathcal{C}(\alpha)$ (the group of contact diffeomorphisms of $(M, \alpha))$ to the group $\operatorname{Diff}_{\mathcal{S}(\alpha)}\left(M \times S^{1}\right)$.

\subsection{Deformations of lcs structures}

If we add a 2-form $\eta_{\epsilon} C^{0}$ close to 0 to a lcs form $\Omega$, the resulting form $\Omega_{\epsilon}=\Omega+\eta_{\epsilon}$ is again non-degenerate. An immediate calculation gives:

$$
d \Omega_{\epsilon}=-\omega \wedge \Omega_{\epsilon}+\left(d \eta_{\epsilon}+\omega \wedge \eta_{\epsilon}\right)=-\omega \wedge \Omega_{\epsilon}+d_{\omega} \eta_{\epsilon}
$$

Hence if $\eta_{\epsilon}$ is $d_{\omega}$ closed, then $\Omega_{\epsilon}$ is a lcs form with $\omega$ as Lee form. For instance take $\eta_{\epsilon}=d_{\omega} \gamma_{\epsilon}$ where $\gamma_{\epsilon}$ is $C^{1}$ close to zero.

To construct general deformations of a lcs form $\Omega$, with Lee form $\omega$, we may look for 2-forms $\eta_{\epsilon} C^{0}$ closed to zero, and closed 1-forms $\rho$ (not necessarily small) such that $d \Omega_{\epsilon}=-(\omega+\rho) \wedge \Omega_{\epsilon}$. In that connection, we note that if $\mathcal{L}_{c s}(M)$ is the set of all lcs forms on a smooth manifold $M$, and $\mathcal{F}^{*}(M)$ the space of differential forms, both with the $C^{\infty}$ topology, $\mathcal{L}_{c s}(M)$ is not an open subset of $\mathcal{F}^{*}(M)$.

Note that if the lcs form $\Omega$ is of first kind and we add to it a non- $d_{\omega}$-exact form, the resulting lcs form is not $d_{\omega}$-exact, hence of the second kind.

We have the following fact:

Theorem 2. Let $(M, \mathcal{S})$ be a compact lcs manifold, and let $\Omega \in \mathcal{S}$ be a representative, with Lee form $\omega$. Then for any $d_{\omega}$ exact 2 -form $\eta_{\epsilon}, C^{0}$ close to zero, the lcs form $\Omega_{\epsilon}=\Omega+\eta_{\epsilon}$ represents a lcs structure equivalent to $\mathcal{S}$.

Hence the non-trivial deformations of lcs structures are parametrized by elements of the second cohomology group $H_{\omega}^{2}(M)$.

\subsection{Lcs on cotangent bundles [12]}

Let $M=T^{*}(N)$ be the total space of the cotangent bundle $\pi: T^{*}(N) \rightarrow N$ over a smooth manifold $N$. Let $\Lambda_{N}$ be the Liouville 1-form on $M$ and $\alpha$ a closed 1-form on $N$, then

$$
\Omega_{\alpha}=d_{\omega} \Lambda_{N}
$$

where $\omega=\pi^{*} \alpha$, is a lcs form on $M$. The conformal structure defined by this lcs form depends only on the cohomology class of $\alpha$. 


\section{The $c \mathcal{A}$-cohomology and the $d_{\omega}$-cohomology}

For any closed 1-form $\omega$ on a smooth manifold $M$, the operator $d_{\omega}$ which assigns to a $p$-form $\gamma$ the $(p+1)$-form

$$
d_{\omega} \gamma=d \gamma+\omega \wedge \gamma
$$

is a coboundary operator, i.e. $d_{\omega} \circ d_{\omega}=0$.

The cohomology of differential forms with this coboundary operator will be denoted by $H_{\omega}^{*}(M)$ and will be called the $d_{\omega}$-cohomology. For more information on this cohomology, see [11] or [19]. For instance, it was proved in [19] that the groups $H_{\omega}^{p}(M)$ are isomorphic to the cohomology groups of $M$ with coefficients in the sheaf $\mathcal{F}_{\omega}(M)$ of germs of smooth functions $f$ on $M$ such that $d_{\omega} f=0$.

In this section, we give another interpretation of the $d_{\omega}$ cohomology.

One associates with a closed 1-form $\omega$ on a smooth manifold $M$ the minimum regular cover $\pi: \tilde{M} \rightarrow M$ over which the 1-form $\omega$ pulls back to an exact 1form. The manifold $\tilde{M}$ is a connected component of the sheaf of germs of smooth functions $f$ on $M$ such that $\omega=d f[10]$.

Let $\lambda: \tilde{M} \rightarrow \mathbb{R}$ be a positive function on $\tilde{M}$ such that

$$
\pi^{*} \omega=d(\ln \lambda) .
$$

It is well known that the group $\mathcal{A}$ of automorphisms of the covering $\tilde{M}$, is isomorphic to the group of periods of $\omega[10]$. We will need the following:

Lemma $1[6]$. For any $\tau \in \mathcal{A}$, the function

$$
(\lambda \circ \tau) / \lambda
$$

is a constant, we denote $c_{\tau}$, independent of the choice of $\lambda$ and

$$
\tau \mapsto c_{\tau}
$$

is a group homomorphism c from $\mathcal{A}$ to the multiplicative group $\mathbb{R}^{+}$of positive real numbers.

For the convenience of the reader, we give here the proof [6].

Proof. Clearly if $\lambda^{\prime}=a \lambda$ for some constant $a, \lambda^{\prime} \circ \tau / \lambda^{\prime}=\lambda \circ \tau / \lambda$.

For any $\tau \in \mathcal{A}$, we have:

$$
d(\ln (\lambda \circ \tau)-\ln \lambda))=\tau^{*} \pi^{*} \omega-\pi^{*} \omega=(\pi \tau)^{*} \omega-\pi^{*} \omega=\pi^{*} \omega-\pi^{*} \omega=0 .
$$

Hence $\ln (\lambda \circ \tau / \lambda)=K$, a constant and $\lambda \circ \tau / \lambda=e^{K}=c_{\tau}$. 
If $\tau, \tau^{\prime} \in \mathcal{A}:$

$$
\begin{gathered}
c_{\tau \tau^{\prime}}=\left(\lambda \circ \tau \tau^{\prime}\right) / \lambda=\left(\left(\lambda \circ\left(\tau \tau^{\prime}\right)\right) /\left(\lambda \circ \tau^{\prime}\right)\right) \cdot\left(\lambda \circ \tau^{\prime}\right) / \lambda \\
\left.=((\lambda \circ \tau) / \lambda) \circ \tau^{\prime}\right) \cdot\left(\left(\lambda \circ \tau^{\prime}\right) / \lambda\right)=((\lambda \circ \tau) / \lambda) \cdot\left(\left(\lambda \circ \tau^{\prime}\right) / \lambda\right)=c_{\tau} \cdot c_{\tau^{\prime}} .
\end{gathered}
$$

The set $\mathcal{F}_{c \mathcal{A}}^{*}(M)$ of all differential forms $\alpha$ on $\tilde{M}$ such that $\tau^{*} \alpha=c_{\tau} \alpha$ for all $\tau \in \mathcal{A}$, is a subcomplex of the de Rham complex of $\tilde{M}$. We denote its cohomology by $H_{c \mathcal{A}}^{*}(M)$ and call it the conformally $\mathcal{A}$-invariant cohomology of $M$. Clearly, if the cohomology class of $\omega$ is trivial, then $H_{c \mathcal{A}}^{*}(M)$ coincides with the de Rham cohomology of $M$.

Remark 1. For any differential form $\alpha$ on $M$, then $U_{\alpha}=\lambda \pi^{*} \alpha \in \mathcal{F}_{c \mathcal{A}}^{*}(M)$ Indeed, for any $\tau \in \mathcal{A}$,

$$
\tau^{*} U_{\alpha}=\lambda \circ \tau \cdot \tau^{*} \pi^{*} \alpha=\frac{\lambda \circ \tau}{\lambda} \cdot \lambda \cdot(\pi \circ \tau)^{*} \alpha=c_{\tau}\left(\lambda \pi^{*} \alpha\right)=c_{\tau} U_{\alpha}
$$

Lemma 2. For any differential form, $\alpha, d_{\omega} \alpha=0$ if and only if $d\left(\lambda \pi^{*} \alpha\right)=0$.

Proof. Suppose $d_{\omega} \alpha=0$. Then: $d\left(\lambda \pi^{*} \alpha\right)=d \lambda \wedge \pi^{*} \alpha+\lambda \pi^{*}(-\omega \wedge \alpha)=d \lambda \wedge \pi^{*} \alpha-$ $\lambda d(\ln \lambda) \wedge \pi^{*} \alpha=0$.

Suppose now $d\left(\lambda \pi^{*} \alpha\right)=0$, and compute:

$\lambda \pi^{*}\left(d_{\omega} \alpha\right)=\lambda \pi^{*} d \alpha+\lambda \pi^{*} \omega \wedge \pi^{*} \alpha=\lambda \pi^{*} d \alpha+\lambda d(\ln \lambda) \wedge \pi^{*} \alpha=d\left(\lambda \pi^{*} \alpha\right)=0$.

Since $\lambda$ is a positive function and $\pi$ is a local diffeomorphism, $d_{\omega} \alpha=0$.

Theorem 3. $H_{c \mathcal{A}}^{*}(M)$ is (non-canonically) isomorphic with $H_{\omega}^{*}(M)$

Proof. The natural homomorphism

$$
H_{\omega}^{*}(M) \rightarrow H_{c \mathcal{A}}^{*}(M) \quad[\alpha] \mapsto\left[\lambda \pi^{*} \alpha\right]
$$

is onto: indeed, let $\beta$ be a form such that $d \beta=0$ and $\tau^{*} \beta=c_{\tau} \beta$ for all $\tau \in \mathcal{A}$. Then:

$$
\tau^{*}(\beta / \lambda)=\tau^{*} \beta / \lambda \circ \tau=\left(c_{\tau} \cdot \beta / \lambda\right) \cdot(\lambda / \lambda \circ \tau)=\beta / \lambda
$$

for all $\tau \in \mathcal{A}$. Hence $\beta / \lambda$ is basic, i.e. there is a form $\alpha$ on $M$ such that $\beta / \lambda=\pi^{*} \alpha$. Since $\beta=\lambda \pi^{*} \alpha$ is closed, $\alpha$ is $d_{\omega}$ closed, by Lemma 2 .

It is also one-to-one: suppose $d_{\omega} \alpha=0$ and $\lambda \pi^{*} \alpha=d \rho$ with $\tau^{*} \rho=c_{\tau} \rho$ for all $\tau \in \mathcal{A}$. Then: rewriting the equations above with $\beta$ replaced by $\rho$, we see that $\rho / \lambda$ is basic, i.e. there is a form $\gamma$ on $M$ such that $\rho / \lambda=\pi^{*} \gamma$.

Let us now compute: $\pi^{*}\left(d_{\omega} \gamma\right)=\pi^{*}(d \gamma+\omega \wedge \gamma)=d(\rho / \lambda)+d \ln \lambda \wedge \rho / \lambda=$ $d \rho / \lambda-d \lambda /(\lambda)^{2} \wedge \rho+(d \lambda / \lambda) \wedge \rho / \lambda=d \rho / \lambda=\pi^{*} \alpha$. 
Since $\pi$ is a covering map, $\alpha=d_{\omega} \gamma$.

In [5], [6], we had already observed that $H_{c \mathcal{A}}(M)$ is a quotient of $H_{\omega}(M)$. We deduce the following well known fact $([11])$

Corollary. If $\omega$ is a non-exact 1 -form on a smooth manifold $M, H_{\omega}^{0}(M)=0$.

Proof. An element of $H_{\omega}^{0}(M) \approx H_{c \mathcal{A}}^{0}(M)$ is represented by a constant $K$ such that $K \circ \tau=K=c_{\tau} K$ for all $\tau \in \mathcal{A}$. Since $\omega$ is not exact, there is a $\tau \in \mathcal{A}$ with $c_{\tau} \neq 1$. Hence $K=0$.

Let $(M, \mathcal{S})$ be a lcs manifold, $\Omega \in \mathcal{S}$ a representative, with Lee form $\omega$. Let $\pi: \tilde{M} \rightarrow M$ be the minimum regular covering of $M$ associated with the 1 -form $\omega$ and let $\lambda: \tilde{M} \rightarrow \mathbb{R}$ be a positive function on $\tilde{M}$ such that

$$
\pi^{*} \omega=d(\ln \lambda) \text {. }
$$

Then $\tilde{\Omega}=\lambda\left(\pi^{*} \Omega\right)$ is a symplectic form on $\tilde{M}$ and its conformal class $\tilde{\mathcal{S}}$ is independent of the choice of $\Omega \in \mathcal{S}$ and of $\lambda$.

Note that given a lcs $\Omega \in \mathcal{S}$, with Lee form $\omega$, the cohomology classes $[\Omega] \in$ $H_{\omega}^{2}(M)$ and $\left[\lambda \pi^{*} \Omega\right] \in H_{c \mathcal{A}}^{2}(M)$ are not invariants of the lcs structure $\mathcal{S}$.

The cohomology groups $H_{c \mathcal{A}}^{*}(M)$ and the $d_{\omega}$ cohomology are "almost" invariants of the lcs structure: since if $\omega$ and $\omega^{\prime}=\omega-d \ln \lambda$ are two Lee forms, then $H_{\omega}(M)$ is isomorphic to $H_{\omega^{\prime}}(M)$, by the isomorphism $\alpha \rightarrow \lambda \alpha$, which unfortunately depends on the choice of $\lambda$. Two such $\lambda$ 's differ by a constant.

\section{Equivalence of lcs structures}

We have the following Moser type result:

Theorem 4. Let $\Omega_{t}$ be a smooth family of lcs forms on a compact manifold $M$. Suppose that for all $t$, the Lee form of $\Omega_{t}$ is the same 1-form $\omega$ and that $\Lambda_{t}=$ $\Omega_{t}-\Omega_{0}$ is $d_{\omega}$ - exact, then there exist a smooth family of diffeomorphisms $\phi_{t}$ with $\phi_{0}=i d$ and a smooth family of functions $f_{t}$ such that $\phi_{t}^{*} \Omega_{t}=f_{t} \Omega_{0}$.

Remark 2. If the smooth family of lcs forms $\Omega_{t}$ has a smooth family $\omega_{t}$ of corresponding Lee forms, and we write $\omega_{t}=\omega_{0}+d \ln u_{t}$ for some positive functions $u_{t}$ (see the beginning of the proof of Theorem 5), then $\Omega_{t}^{\prime}=u_{t} \Omega_{t}$ has $\omega_{0}$ as Lee form for all $t$. Hence assuming $\Lambda_{t}^{\prime}=\Omega_{t}^{\prime}-\Omega_{0}^{\prime}$ to be $d_{\omega_{0}}$-exact, yields that $\Omega_{t}$ represent equivalent lcs structures for all t.

Proof. By assumption, $\partial / \partial t\left(\Omega_{t}\right)$ is $d_{\omega}$ exact for all t. A result of [12], (Lemma 1.9) asserts that there exists a smooth family of 1-forms $\eta_{t}$ such that

$$
\partial / \partial t\left(\Omega_{t}\right)=d_{\omega} \eta_{t}
$$


The argument used to find a smooth lifting of $d_{\omega}$-coboundaries is the same as in [1], (Lemma II.2.2), which is an application of Grothendieck's theory of nuclear topological vector spaces. This replaces the Hodge-de Rham theorem in Moser's theorem for symplectic forms [17].

Let $\tilde{\Omega}_{t}=\lambda \pi^{*} \Omega_{t}$, where $\pi: \tilde{M} \rightarrow M$ is the minimum regular cover and $\lambda$ is such that $\pi^{*} \omega=d \ln \lambda$. We define a smooth family of vector fields $X_{t}$ on $\tilde{M}$ by:

$$
i\left(X_{t}\right) \tilde{\Omega}_{t}=-\lambda \pi^{*} \eta_{t}
$$

Since $d\left(\lambda \pi^{*} \eta_{t}\right)=\lambda \pi^{*} d_{\omega} \eta_{t}$, we have:

$$
L_{X_{t}} \tilde{\Omega}_{t}+\partial / \partial t\left(\tilde{\Omega}_{t}\right)=0
$$

We claim that $X_{t}$ is complete. Hence it defines a smooth family of diffeomorphisms $\psi_{t}$ of $\tilde{M}$ such that $\psi_{t}^{*} \tilde{\Omega}_{t}=\tilde{\Omega}_{0}$.

This argument is Moser's standard path method [17].

To prove that $X_{t}$ is complete, it is enough to show that it is basic, i.e., there is a family of vector fields $Y_{t}$ on $M$ such that $\pi_{*} X_{t}=Y_{t}$. Since $M$ is compact, $Y_{t}$ is integrable, and so will be $X_{t}$.

For any $\tau \in \mathcal{A}$, we easily see that:

$$
\tau^{*} \tilde{\Omega}_{t}=c_{\tau} \tilde{\Omega}_{t},
$$

and

$$
\tau^{*}\left(\lambda \pi^{*} \eta_{t}\right)=c_{\tau}\left(\lambda \pi^{*} \eta_{t}\right)
$$

We therefore have:

$$
\begin{gathered}
\left.\left.-c_{\tau} i\left(X_{t}\right) \tilde{\Omega}_{t}\right)=\tau^{*}\left(\lambda \pi^{*} \eta_{t}\right)=-\tau^{*}\left(i\left(X_{t}\right) \tilde{\Omega}_{t}\right)=-i\left((\tau)^{-1}\right)_{*} X_{t}\right)\left(\tau^{*} \tilde{\Omega}_{t}\right) \\
\left.\left.=-i\left((\tau)^{-1}\right)_{*} X_{t}\right)\left(c_{\tau} \tilde{\Omega}_{t}\right)=-c_{\tau} i\left((\tau)^{-1}\right)_{*} X_{t}\right)\left(\tilde{\Omega}_{t}\right)
\end{gathered}
$$

Hence

$$
\left.\left.c_{\tau} i\left((\tau)^{-1}\right)_{*} X_{t}\right)\left(\tilde{\Omega}_{t}\right)=c_{\tau} i\left(X_{t}\right) \tilde{\Omega}_{t}\right) .
$$

Since $c_{\tau} \neq 0$, we have: $\left.i\left((\tau)^{-1}\right)_{*} X_{t}\right)\left(\tilde{\Omega}_{t}\right)=i\left(X_{t}\right) \tilde{\Omega}_{t}$. Therefore $\left.\left((\tau)^{-1}\right)_{*} X_{t}\right)=X_{t}$.

Let now $\phi_{t}$ be the family of diffeomorphisms of $M$ covered by $\psi_{t}$, i.e. $\pi \circ \psi_{t}=$ $\phi_{t} \circ \pi$, then $\psi_{t}^{*} \tilde{\Omega}_{t}=\left(\lambda_{t} \circ \psi_{t}\right) \cdot \pi^{*}\left(\phi_{t}^{*} \Omega_{t}\right)=\lambda_{0} \pi^{*} \Omega_{0}$. Hence $\pi^{*}\left(\phi_{t}^{*} \Omega_{t}\right)=\left(\lambda_{0} /\left(\lambda_{t} \circ\right.\right.$ $\left.\left.\phi_{t}\right)\right) \pi^{*} \Omega_{0}$. For all $\tau \in \mathcal{A}$, we have:

$$
\left(\lambda_{0} /\left(\lambda_{t} \circ \phi_{t}\right)\right) \pi^{*} \Omega_{0}=\pi^{*}\left(\phi_{t}^{*} \Omega_{t}\right)=\tau^{*} \pi^{*}\left(\phi_{t}^{*} \Omega_{t}\right)=\left(\left(\lambda_{0} /\left(\lambda_{t} \circ \phi_{t}\right) \circ \tau\right) \pi^{*} \Omega_{0} .\right.
$$

Therefore, $\left(\lambda_{0} / \lambda_{t} \circ \phi_{t}\right)$ is invariant by all $\tau \in \mathcal{A}$, hence $\left(\lambda_{0} / \lambda_{t} \circ \phi_{t}\right)=f_{t} \circ \pi$ for some function $f_{t}$ on $M$. We thus get that $\pi^{*}\left(\phi_{t}^{*} \Omega_{t}\right)=\pi^{*}\left(f_{t} \Omega_{0}\right)$, and hence $\phi_{t}^{*} \Omega_{t}=f_{t} \Omega_{0}$.

This finishes the proof of Theorem 4 . 
Exactly like in Moser's theorem in Symplectic Geometry [17], there are examples in which we get smooth liftings of the coboundaries $\Lambda_{t}$ without using the deep lemma (which is an application of Grothendieck's theory of topological vector spaces). The most trivial example is provided by Theorem 2: if $\eta_{\epsilon}=d_{\omega} \gamma_{\epsilon}$, then $\Lambda_{t}=d_{\omega}\left(t \gamma_{\epsilon}\right)$

In the following situation, we also have an immediate smooth lifting of the coboundaries $\Lambda_{t}$.

Theorem 5. Let $\Omega_{t}$ be a smooth family of lcs forms on a compact manifold $M$, with a smooth family $\omega_{t}$ of Lee forms having a fixed de Rham cohomology, i.e. $\left[\omega_{0}\right]=\left[\omega_{t}\right], \forall t$, and such that there exists a smooth family $\theta_{t}$, with $\Omega_{t}=d \theta_{t}+\omega_{t} \wedge \theta_{t}$, then the lcs forms $\Omega_{t}$ define equivalent lcs structures.

Proof. There is a smooth family of positive functions $u_{t}$ on $M$ with $\omega_{t}=\omega_{0}+$ $d \ln \left(u_{t}\right)$ and $u_{0}=1$. Indeed, since $(\partial / \partial t)\left(\omega_{t}\right)$ is exact, there is a smooth family of positive functions $v_{t}$ such that $(\partial / \partial t)\left(\omega_{t}\right)=d \ln \left(v_{t}\right)$. Use for instance the Hodgede Rham decomposition theorem. Now integrate both side and set $u_{t}=\int_{0}^{t}\left(v_{s}\right) d s$.

Let $\pi: \tilde{M} \rightarrow M$ be the minimum cover associated with $\omega_{0}$, and let $\lambda_{0}: \tilde{M} \rightarrow \mathbb{R}$ be a positive function such that $\pi^{*} \omega_{0}=d \ln \lambda_{0}$. Then $\pi^{*} \omega_{t}=d \ln \lambda_{0}+d \ln \left(u_{t} \circ \pi\right)=$ $d \ln \lambda_{t}$ with $\lambda_{t}=\lambda_{0} \cdot\left(u_{t} \circ \pi\right)$. We have:

$$
\tilde{\Omega}_{t}=\lambda_{t} \pi^{*} \Omega_{t}=\lambda_{t} \pi^{*}\left(d \theta_{t}\right)+\lambda_{t} d \ln \lambda_{t} \wedge \pi^{*} \theta_{t}=d\left(\lambda_{t} \pi^{*} \theta_{t}\right) .
$$

Setting $\partial / \partial t\left(\lambda_{t} \pi^{*} \theta_{t}\right)=\rho_{t}$, we define a smooth family of vector fields $X_{t}$ on $\tilde{M}$ by:

$$
i\left(X_{t}\right) \tilde{\Omega}_{t}=-\rho_{t}
$$

We have:

$$
L_{X_{t}} \tilde{\Omega}_{t}+\partial / \partial t\left(\tilde{\Omega}_{t}\right)=0 .
$$

We claim that $X_{t}$ is complete. Hence it defines a smooth family of diffeomorphisms $\psi_{t}$ of $\tilde{M}$ such that $\psi_{t}^{*} \tilde{\Omega}_{t}=\tilde{\Omega}_{0}$.

From here proceed like in the proof of Theorem 3 .

Remark 3. Let $u_{t}$ be a smooth family of positive functions such that $\omega_{t}=$ $\omega_{0}+d \ln u_{t}$. Then $\Omega_{t}^{\prime}=u_{t} \Omega_{t}$ has $\omega_{0}$ as Lee form for all $t$. Moreover setting $\theta_{t}^{\prime}=u_{t} \theta_{t}$, we have:

$d_{\omega_{0}}\left(\theta_{t}^{\prime}\right)=u_{t} d \theta_{t}+\frac{d u_{t}}{u_{t}} \wedge\left(u_{t} \theta_{t}\right)+\omega_{0} \wedge u_{t} \theta_{t}=u_{t}\left(d \theta_{t}+\left(d \ln u_{t}+\omega_{0}\right) \wedge \theta_{t}\right)=u_{t} \Omega_{t}=\Omega_{t}^{\prime}$.

Hence $\Omega_{t}^{\prime}=d_{\omega_{0}}\left(\theta_{t}^{\prime}\right)$. The coboundary $\Lambda_{t}^{\prime}=\Omega_{t}^{\prime}-\Omega_{0}^{\prime}$ has the smooth lifting $d_{\omega_{0}}\left(\theta_{t}^{\prime}-\theta_{0}^{\prime}\right)$.

Proof of Theorem 1. Theorem 1 is a consequence of Theorem 5 since two contact forms $\alpha, \alpha^{\prime}$ define the same contact structure if $\alpha^{\prime}=w \alpha$, with $w$ a smooth positive 
function. Now set $\alpha_{t}=\exp (t \ln (w)) \alpha$. The family of lcs forms is $\Omega_{t}=d \theta_{t}+\omega \wedge \theta_{t}$ with $\theta_{t}=p_{1}^{*} \alpha_{t}$.

The mapping $\rho: \operatorname{Diff}_{\mathcal{C}(\alpha)}(M) \rightarrow \operatorname{Diff}_{\mathcal{S}(\alpha)}\left(M \times S^{1}\right)$ comes from the proof. For $h \in \operatorname{Diff}_{\mathcal{C}(\alpha)}(M), h^{*} \alpha=w . \alpha$, then the diffeomorphism $\phi_{1}$ above obtained using $\Omega_{t}=d \theta_{t}+\omega \wedge \theta_{t}$, with $\theta_{t}=p_{1}^{*} \alpha_{t}$ and $\alpha_{t}=\exp (t \cdot \ln (w)) \alpha$, takes $\Omega_{1}$ to $a \Omega_{0}$. Taking a path from $h \alpha$ to $\alpha$, which does not reverse the first one, for instance $\alpha_{t}^{\prime}=(t+(1-t) h) \alpha, \theta_{t}^{\prime}=p_{1}^{*} \alpha_{t}^{\prime}$ and $\Omega_{t}^{\prime}=d \theta_{t}^{\prime}+\omega \wedge \theta_{t}^{\prime}$, get a diffeomorphism $\phi_{1}$ taking $\Omega_{0}$ back to a multiple of $\Omega_{1}$. Now set $\rho(h)=\phi_{1} \circ \psi_{1}$.

\section{Invariants of lcs structures}

Given a lcs manifold $(M, \mathcal{S})$, we have considered the following objects attached to $\mathcal{S}$ :

1. The cohomology class of the Lee form $\omega$ of any representative lcs form $\Omega \in \mathcal{S}$. We saw that this is an invariant $\mathcal{L}_{\mathcal{S}}$, we called the Lee class of $\mathcal{S}$. The group $\mathcal{A}$ of periods of $\omega$ is an object depending only on the conformal class $\mathcal{S}$.

2 . We considered the minimum cover of $M$ which has a group of deck transformations isomorphic with the group $\mathcal{A}$ of periods of $\omega$ as group of automorphisms, and the $c \mathcal{A}$ cohomology.

In Proposition 1, we gather other invariants built using the automorphisms of the lcs structure.

If $\mathcal{G}$ is a Lie algebra and $K$ is a $\mathcal{G}$-module, we denote by $H^{*}(\mathcal{G}, K)$, the cohomology of $\mathcal{G}$ with coefficients in $K$ [14]. This is the cohomology of the complex $\left(C^{*}(\mathcal{G}, K), \delta\right)$ where $p$-cochains are $p$-linear alternating mappings on $\mathcal{G}$ with values in $K$ and the coboundary operator is given by:

$$
\begin{aligned}
\partial f\left(X_{1}, \ldots, X_{p+1}\right)= & \sum_{i}(-1)^{i+1} X_{i} \cdot f\left(X_{1}, \ldots, \hat{X}_{i}, \ldots X_{p+1}\right) \\
& +\sum_{i \leq j}(-1)^{i+j} f\left(\left[X_{i}, X_{j}\right], \ldots, \hat{X}_{i}, \ldots \hat{X}_{j}, \ldots\right) .
\end{aligned}
$$

We also consider the cohomology $H^{*}(G, K)$ of an (abstract) group $G$ into a $G$-module $K$ [13]. The $p$-cochains now are mappings from $G^{p}$ to $K$ and the coboundary operator $\delta$ is given by

$$
\begin{aligned}
\delta g\left(a_{0}, \ldots, a_{p}\right)= & a_{0} \cdot c\left(a_{1}, \ldots, a_{p}\right)-\left(\sum_{i}(-1)^{i} c\left(a_{0}, \ldots, a_{i} a_{i+1}, \ldots a_{p}\right)\right) \\
& +(-1)^{p+1} c\left(a_{0}, \ldots, a_{p-1}\right) .
\end{aligned}
$$

$H^{1}(G, K)$ is the quotient of derivations (1-cocycles) by inner derivations (coboundaries). Recall that derivations are maps $d: G \rightarrow K$ such that $d(g h)=$ $g . d(h)+d g$ and an inner derivation is a map $v: G \rightarrow K$ such that there exists $k \in K$ such that $v(g)=g . k-k$. 
$H^{1}(\mathcal{G}, K)$ is the quotient of the space of linear maps $v: \mathcal{G} \rightarrow K$ such that $u([X, Y])=X . u(Y)-Y . u(X)$ (1-cocycles), modulo (the coboundaries) consisting of linear maps $v$ such that there exists $k \in K$ with $v(X)=X . k$, for all $X, Y \in \mathcal{G}$.

Proposition 1. Let $\mathcal{S}$ be a lcs structure on $M$, and $\Omega \in \mathcal{S}$ with Lee form $\omega$.

1. The map $D_{\Omega}: \operatorname{Diff}_{\mathcal{S}}(M) \rightarrow C^{\infty}(M), \phi \mapsto \ln \left(f_{\phi^{-1}}\right)$, if $\phi^{*} \Omega=f_{\phi} \Omega$ is a 1-cocycle on $\operatorname{Diff}_{\mathcal{S}}(M)$ whose cohomology class $a_{\mathcal{S}} \in H^{1}\left(\operatorname{Diff}_{\mathcal{S}}(M), C^{\infty}(M)\right)$ is independent of the choice of $\Omega \in \mathcal{S}$, i.e. an invariant of $\mathcal{S}$.

2. The map $d_{\Omega}: \mathcal{X}_{\mathcal{S}}(M) \rightarrow C^{\infty}(M), X \mapsto u_{\Omega}(X)$, where $L_{X} \Omega=\left(u_{\Omega}(X)\right) \Omega$, is a 1-cocycle, whose cohomology class $b_{\mathcal{S}} \in H^{1}\left(\mathcal{X}_{\mathcal{S}}(M), C^{\infty}(M)\right)$ is independent of the choice of $\Omega \in \mathcal{S}$, i.e., an invariant of $\mathcal{S}$.

3. The map $\hat{\omega}: \mathcal{X}_{\mathcal{S}}(M) \rightarrow C^{\infty}(M), X \mapsto \omega(X)$ is a 1-cocycle, whose cohomology class $c_{\mathcal{S}} \in H^{1}\left(\mathcal{X}_{\mathcal{S}}(M), C^{\infty}(M)\right)$ is independent of the choice of $\Omega \in \mathcal{S}$, i.e. an invariant of $\mathcal{S}$.

4. The sum $d_{\Omega}+\hat{\omega}$ is a 1-cocycle on $\mathcal{X}_{\mathcal{S}}(M)$ with values in $\mathbb{R}$, hence a homomorphism l, called the extended Lee homomorphism, an invariant of $\mathcal{S}$.

5. Suppose $M$ is compact and fix a riemannian metric. For each $h \in \operatorname{Diff}_{\mathcal{S}}(M)$ (not even homotopic to the identity) $h^{*} \omega-\omega$ is an exact 1-form. Let $u_{h}$ be the unique function provided by the Hodge decomposition of $h^{*} \omega-\omega$ such that $h^{*} \omega-\omega=$ $d u_{h}$.

For $h, h^{\prime} \in \operatorname{Diff}_{\mathcal{S}}(M)$ :

$$
\left(h, h^{\prime}\right) \mapsto u_{h} \circ h^{\prime}+u_{h^{\prime}}-u_{h h^{\prime}}
$$

is a 2 -cocycle $K_{\omega}$ with values in $\mathbb{R}$. Its cohomology class in $H^{2}\left(\operatorname{Diff}_{\mathcal{S}}(M), \mathbb{R}\right)$ is an invariant $\mathcal{K}_{\mathcal{S}}$ of $\mathcal{S}$.

Statements 1, and 2 have been observed in [2]. The statement 3 is obvious, since the coboundary operator in the Gelfand-Fucks cohomology (cohomology on Lie algebras of vector fields) is the same as in the de Rham cohomology.

The class $c_{\mathcal{S}}$ may be called the Gelfand-Fucks class of $\mathcal{S}$.

Statement 4 was proved by Vaisman [18]. See also [6].

Statement 5 was proved in [8]. The Hodge-de Rham theory gives a smooth lifting of de Rham coboundaries: i.e. any exact $p$-form $\theta$ determines uniquely a $(p-1)$-form $\alpha$ such that $\theta=d \alpha$ as follows: let $\delta$ be the codifferential, and $G$ the Green operator defined by a riemannian metric, then $\alpha=\delta G(\theta)$. Here the function $u_{h}$ is $u_{h}=\delta\left(G\left(h^{*} \omega-\omega\right)\right)$. See for instance [3].

Remark 4. We can define similar invariants using objects with compact support, and denote them by $a_{\mathcal{S}}^{c}, b_{\mathcal{S}}^{c}, c_{\mathcal{S}}^{c}$.

Definition. The structure $\mathcal{S}$ is called inessential if there exists $\Omega_{*} \in \mathcal{S}$ such that $G_{\Omega_{*}}(M)=\operatorname{Diff}_{\mathcal{S}}(M)$. The structure $\mathcal{S}$ is called essential otherwise.

The following fact was observed in [4]: 
Proposition 2. Let $(M, \mathcal{S})$ be a lcs manifold. Then $\mathcal{S}$ is inessential iff $a_{\mathcal{S}}=0$.

The connection between these invariants, and the problem of essentiality, and globality of locally conformal structure is given by the following:

Theorem 6. Let $(M, \mathcal{S})$ be a lcs manifold.

1. If $a_{\mathcal{S}}=0$, then $\mathcal{S}=\mathcal{O}$. Furthermore, the Lee homomorphism is trivial, and the structure $\mathcal{S}$ is of the second kind. Thus inessential structures are of the second kind. This also says that if $\mathcal{S}$ is of the first kind, then $a_{\mathcal{S}} \neq 0$.

2. If $M$ is compact, then $\mathcal{S}=\mathcal{O}$ implies that $a_{\mathcal{S}}=0$.

3. The Gelfand-Fucks class $c_{\mathcal{S}}$ vanishes iff the Lee class $\mathcal{L}_{\mathcal{S}}$ does.

4. If $M$ is compact, the vanishing of one of the four classes $a_{\mathcal{S}}, b_{\mathcal{S}}, c_{\mathcal{S}}, \mathcal{L}_{\mathcal{S}}$, implies the vanishing of the remaining three classes.

We will need the following "local transitivity" result. Lefebvre's [16] proved it away from the zeros of the Lee form. Since for any point, the lcs structure can be represented by a lcs form with Lee form not vanishing at that point, Lefebvre's argument applies. For the convenience of the reader, we rewrote it in our style.

Theorem 7. Let $(M, \mathcal{S})$ be a lcs manifold of dimension $2 n$. For each $x \in M$, there exist $2 n$ vector fields $V_{j}^{x} \in \mathcal{X}_{\mathcal{S}}(M)$ with arbitrarily small compact support in an open neighborhood of $x$ and such that $\left\{V_{j}^{x}(x)\right\}_{j=1, \ldots, 2 n}$ form a basis of the tangent space $T_{x} M$.

Proof. 1. For each point $x \in M$, there is $\Omega \in \mathcal{S}$, with Lee form $\omega$ such that $\omega(x) \neq 0$. Indeed, if the Lee form $\omega$ of $\Omega \in \mathcal{S}$ vanishes at $x$, consider a contractible neighborhood $U$ of $x$ at which $\left.\omega\right|_{U}=d \ln (\lambda)$, and choose a smooth positive function $\rho$, constant outside of $U$ with $d \rho(x) \neq 0$ and $d \ln \lambda \neq d \ln \rho$ on a neighborhood of $x$. The form $\rho \Omega \in \mathcal{S}$ and has Lee form $\omega^{\prime}=\omega-d \ln (\rho)$. The new Lee form does not vanish at $x$ (and in a neighborhood).

2. Any function $u$ on an open set $U$ where $f \Omega_{\mid U}$ is symplectic defines a vector field $X_{u}$ on $U$ by the equation:

$$
\left.i\left(X_{u}\right) f \Omega_{\mid} U\right)=d(f u) .
$$

A direct calculation shows that $\left.L_{X_{u}} \Omega_{\mid} U\right)=\left(-X_{u} \cdot \ln f\right) \Omega[18]$.

3. The form $\Omega \in \mathcal{S}$ above has a Lee form $\omega$ not vanishing on an open neighborhood $V \subset U$ of $x$. Hence, there are local coordinates $\left(x_{1}, \ldots x_{n}, y_{1}, \ldots, y_{n}\right)$ defined on a smaller neighborhood $V_{1}$ of $x$ such that $y_{1} \neq 0$, and

$$
\left.\Omega\right|_{U_{1}}=y_{1}\left(\sum_{k=1}^{n} d x_{k} \wedge d y_{k}\right) .
$$

Let $\mu$ be a smooth function, supported in $V_{2}$ and which is equal to 1 on a closed neighborhood $F$ of $x$, where $F \subset V_{2} \subset V_{1}$. 
We define $2 \mathrm{n}$ vector fields by:

$$
i\left(Y_{1}\right)\left(\frac{1}{y_{1}} \Omega_{\mid V_{1}}\right)=d\left(\mu \frac{y_{1}^{2}}{y_{1}}\right)=d\left(\mu y_{1}\right)
$$

and for $j=2, \ldots, n$,

$$
i\left(Y_{j}\right)\left(\frac{1}{y_{1}} \Omega_{\mid V_{1}}\right)=d\left(\mu \frac{y_{j}}{y_{1}}\right) .
$$

For $j=1, \ldots, n$ define $X_{j}$ by:

$$
i\left(X_{j}\right)\left(\frac{1}{y_{1}} \Omega_{\mid V_{1}}\right)=d\left(\mu \frac{x_{j}}{y_{1}}\right) .
$$

Then $X_{i}, Y_{i}$ are smooth vector fields on $M$ with compact support in $V_{1}$, which all belong to $\mathcal{X}_{\mathcal{S}}(M)_{c}$.

Let us note $e_{j}=\partial / \partial x_{j}$ and $e_{j}^{\prime}=\partial / \partial y_{j}$, then on $F$, we have

$$
\begin{gathered}
Y_{1}=e_{1}, \quad Y_{j}=\frac{1}{y_{1}} e_{j}-\frac{y_{j}}{y_{1}^{2}} e_{1}, \quad j=2, \ldots, n \\
X_{j}=-\frac{1}{y_{1}} e_{j}^{\prime}-\frac{x_{j}}{y_{1}^{2}} e_{1}, \quad j=1, \ldots, n .
\end{gathered}
$$

Writing that $\sum_{i=1}^{n}\left(a_{i} X_{i}+b_{i} Y_{i}\right)=0$, gives immediately that $b_{i}=0$ and $a_{i}=0$, i.e. these vector fields are linearly independent near $x$.

Proof of Theorem 6. 1. Suppose that $a_{\mathcal{S}}=0$, that is $\mathcal{S}$ is inessential (Proposition 2 ). Let $\Omega_{*} \in \mathcal{S}$ with $\operatorname{Diff}_{\mathcal{S}}(M)=G_{\Omega_{*}}(M)$, and let $\omega_{*}$ be the corresponding Lee form. It follows that

$$
\mathcal{X}_{\mathcal{S}}(M)_{c}=\mathcal{X}_{\Omega_{*}}(M)_{c}
$$

Let us now show that $\omega_{*}=0$.

For each $x \in M$, and any tangent vector $\xi \in T_{x} M$, we want to show that $\omega_{*}(x)(\xi)=0$. By Theorem $7, \xi=\sum_{j=1}^{2 n} c_{j}(x) V_{j}^{x}(x)$. Extend now the coefficients $c_{j}(x)$ into smooth functions $c_{j}$ with compact support near $x$. We get a smooth vector field with compact support $V=\sum_{j=1}^{2 n} c_{j} V_{j}^{x}$, which coincides with $\xi$ at $x \in M$. Therefore,

$$
\omega_{*}(x)(\xi)=\omega_{*}(x)(V(x))=\left(\omega_{*}(V)\right)(x)=\sum_{j=1}^{2 n}\left(c_{j} \omega_{*}\left(V_{j}^{x}\right)\right)(x) .
$$

Since $\left.V_{j}^{x} \in \mathcal{X}_{\mathcal{S}}(M)_{c}=\mathcal{X}_{\Omega_{*}}(M)_{c}, \omega_{*}\left(V_{j}^{x}\right)\right)$ is a constant function (see Remark 5.3) with compact support, and hence identically zero. This proves that $\omega_{*}(x)=0$.

This implies that $\mathcal{S}=\mathcal{O}$. 
Since the Lee homomorphism can be computed using $\Omega_{*}$ and $\omega_{*}$, we see that

$$
l=\hat{\omega}_{*}=0 .
$$

This implies that the structure is of the second kind. Indeed, if $\Omega$ is any representative of $\mathcal{S}$ with Lee form $\omega$ and $X \in \mathcal{X}_{\Omega}(M)$, then $l(X)=\omega(X)=0$.

2. If $\mathcal{S}=\mathcal{O}$, there is a symplectic form $\Omega \in \mathcal{S}$. If $\phi \in \operatorname{Diff}_{\mathcal{S}}(M)$, then $\phi^{*} \Omega=f \Omega$. By the classical theorem of Libermann (see [6]), $f$ is a constant, provided that the dimension of $M$ is at least 4 , (which is assumed here) and if $M$ is compact, this constant must be 1 . This follows from the fact that $\int_{M} \phi^{*} \Omega^{n}=f^{n} \int_{M} \Omega^{n}$ and by the formula of change of variable, we have equality with $\int_{M} \Omega^{n}$. Hence $f=1$ and therefore $a_{\mathcal{S}}=0$.

3. It is clear that $[\omega]=0$ implies that $[\hat{\omega}]=0$. Conversely, suppose there exists a smooth function $u$ such that $\omega(X)=X . u=d u(X)$ for all $X \in \mathcal{X}_{\mathcal{S}}(M)$. We show that indeed $\omega(\xi)=d u(\xi)$ for all vector fields $\xi$, i.e that $\omega=d u$. For each point $x \in M$, we need to show that $\omega(\xi)(x)=(d u(\xi)(x))$.

As above, we consider the vector field $V=\sum_{j=1}^{2 n} c_{j} V_{j}^{x}$, which is equal to $\xi$ at $x$. Then, like above: $\omega(\xi)(x)=\sum_{j=1}^{2 n}\left(c_{j} \omega\left(V_{j}^{x}\right)\right)(x)=\sum_{j=1}^{2 n}\left(c_{j} d u(x)\left(V_{j}^{x}\right)\right)=$ $d u(x)\left(\sum_{j=1}^{2 n} c_{j} V_{j}^{x}\right)=d u(x)(V)=d u(x)(\xi)$. Therefore the de Rham class of $\omega$ is trivial.

4. In the compact case $\left(a_{\mathcal{S}}=0\right) \Leftrightarrow(\mathcal{S}=\mathcal{O})$ and $\left(a_{\mathcal{S}}=0\right) \Leftrightarrow\left(b_{\mathcal{S}}=0\right)$.

We also have that in general, $\left(\mathcal{S}=\mathcal{O} \Leftrightarrow\left(\mathcal{L}_{\mathcal{S}}=0\right)\right.$ and $\left(c_{\mathcal{S}}=0\right) \Leftrightarrow\left(\mathcal{L}_{\mathcal{S}}=0\right)$

Putting these facts together, yields the last assertion of Theorem 5 .

Remarks. 1. If $M$ is not compact, $\mathcal{S}=0$ does not imply that $a_{\mathcal{S}}=0$. Take for instance the global conformal symplectic structure defined by the standard symplectic form on $\mathbb{R}^{2 n}$, and more generally non-compact manifolds with complete Liouville vector fields, like Stein manifolds [4].

2 . The vanishing of the compactly supported invariant $a_{\mathcal{S}}^{c}$ also implies that $\mathcal{S}=0$. This was proved in $[12]$.

\section{Concluding remarks and questions}

1. The mapping $L: \mathcal{L}_{c s}(M) \rightarrow \mathcal{F}^{1}(M)$ assigning to a lcs form its Lee form is not continuous in the $C^{0}$ topology. Indeed if $u$ is a smooth function which is $C^{0}$ close to 1 and $C^{1}$ far from 0 , then the Lee forms of $u \Omega$ and $\Omega$, are far apart. How about the continuity for the $C^{\infty}$ topology?

If $M$ has a complex structure $J$ and a hermitian metric $g$ such that the lcs form $\Omega$ is given by $\Omega(X, Y)=g(X, J Y)$ ( $M$ is said to be a locally conformal Kaehler manifold), then $L$ is continuous for the $C^{\infty}$ topology. Indeed in that case we have an explicit formula for $L(\Omega)[9]$ :

$$
L(\Omega)=\frac{1}{n-1}(\delta \Omega \circ J) .
$$


Here $\delta$ is the codifferential with respect to the metric $g$, and $2 n$ is the dimension of $M$.

2. The Lee homomorphism $l: \mathcal{X}_{\mathcal{S}}(M) \rightarrow \mathbb{R}$ can be integrated into a homomorphism $\mathcal{L}: \operatorname{Diff}_{\mathcal{S}}(M)_{+} \rightarrow \mathbb{R} / \Delta$ (where $\Delta$ is some countable subgroup of $\mathbb{R}$ ), and $\operatorname{Diff}_{\mathcal{S}}(M)_{+}$is the group of automorphisms of $\mathcal{S}$ which admit a lift to the minimal regular cover $\tilde{M}[6]$.

If $\alpha$ is a contact form on a compact manifold $M$, we constructed in Theorem 1 a map $\rho: \operatorname{Diff}_{\mathcal{C}(\alpha)}(M) \rightarrow \operatorname{Diff}_{\mathcal{S}(\alpha)}\left(M \times S^{1}\right)_{+}$. Composing $\rho$ with the extended global Lee homomorphism, we get a map:

$$
\mu=\mathcal{L} \circ \rho: \operatorname{Diff}_{\mathcal{C}(\alpha)}(M) \rightarrow \mathbb{R} / \Delta .
$$

This map is not a group homomorphism. This allows us to define a 2-cocycle $\eta$ on the the group $\operatorname{Diff}_{\mathcal{C}(\alpha)}(M)$ :

$$
\eta(\phi, \psi)=\rho(\phi) \cdot \rho(\psi) \cdot(\rho(\phi \psi))^{-1}
$$

for all $\phi, \psi \in \operatorname{Diff}_{\mathcal{C}(\alpha)}(M)$.

What is the meaning of that cocycle?

\section{References}

[1] A. Banyaga, Sur la structure du groupe de diffeomorphismes qui preservent une forme symplectique, Comment. Math. Helv. 53 (1978), 174-227.

[2] A. Banyaga, Invariants of contact structures and transversally oriented foliations, Annals of Global Analysis and Geometry 14 (1996), 427-441.

[3] A. Banyaga, The structure of classical diffeomorphism groups, Mathematics and its applications no 400, Kluwer Academic Publisher, 1997.

[4] A. Banyaga, On essential conformal groups and a conformal invariant, Journal of Geometry 68 (2000), 10-15.

[5] A. Banyaga, Quelques invariants des structures localement conformement symplectiques, C. R. Acad. Sci. Paris 332 Serie 1 (2001), 29-32.

[6] A. Banyaga, A geometric integration of the extended Lee homomorphism, Journal of Geometry and Physics 39 (2001), 30-44.

[7] A. Banyaga, An introduction to symplectic geometry, in: M. Audin, J. Lafontaine (eds), Holomorphic Curves in symplectic geometry, Progress in Math 117, Birkhäuser, 1994, 1740.

[8] A. Banyaga and R. Urwin, Sur la cohomologie du groupe des diffeomorphismes, C. R. Acad. Sci. Paris 294 (1982), 625-627.

[9] S. Dragomir and L. Ornea, Locally conformal Kaehler geometry, Progress in Math. 155, Birkhäuser, 1998.

[10] C. Godbillon, Elements de topologie algebriques, Hermann, Paris, 1971.

[11] F. Guerida and A. Lichnerowicz, Geometrie des algebres de Lie locales de Kirillov, J. Math. Pures et Appl. 63 (1984), 407-484.

[12] S. Haller and T. Rybicki On the group of diffeomorphisms preserving a locally conformal symplectic structure, Ann. Global Anal. and Geom. 17 (1999) 475-502.

[13] P. Hilton and U. Stammback, A course in homological algebra, Springer Graduate Texts in Math., Springer, 1971. 
[14] G. Hochschild and J.-P. Serre, Cohomology of Lie algebras, Ann. Math. 57 (2)(1953), 591603.

[15] H. C. Lee, A kind of even-dimensional differential geometry and its application to exterior calculus, Amer. J. Math. 65 (1943), 433-438.

[16] J. Lefebvre, Propriétés du groupe de transformations conformes et du groupe des automorphismes d'une variété localement conformement symplectique, C. R. Acad. Sci. Paris $\mathbf{2 6 8}$ Serie A (1969), 717-719.

[17] J. Moser, On the volume element of a manifold, Trans. Amer. Math. Soc. 120 (1965), 286-294.

[18] I. Vaisman, Locally conformal symplectic manifolds, Inter. J. Math. and Math. Sci. 8 no 3 (1983), 521-536.

[19] I. Vaisman, Remarkable operators and commutation formulas on locally conformal Kaehler manifolds, Compositio Math. 40 (1980), 227-259.

Augustin Banyaga

The Pennsylvania State University

Department of Mathematics

225 Mc Allister Building

University Park

PA 16803

U.S.A.

e-mail: banyaga@math.psu.edu

(Received: May 28, 2001) 